\title{
Thanks to all those who reviewed for Reproductive Health in 2015
}

José M. Belizán ${ }^{1 *}$, Sunni Mumford² and João Paulo Souza ${ }^{3}$

\section{Contributing reviewers}

Those devoted to medical science have, as their main purpose, the ambition to improve the quality of life of the population. The editorial team of Reproductive Health is mainly devoted to improving reproductive health, particularly for those populations with major needs.

A peer-reviewed journal would not survive without the generous time and insightful comments of the reviewers, whose efforts often go unrecognized. They provide their valuable time without receiving any personal profit to guarantee the quality of research findings. A clear demonstration of generosity, the foundations science was built upon. Final editorial decisions are greatly facilitated by the deeper technical knowledge, scientific insights, understanding of social consequences, and passion that reviewers bring to our deliberations. For these reasons, the Editors-in-Chief and staff of Reproductive Health warmly thank the reviewers of 2015 whose comments helped to shape the journal, for their invaluable assistance with review of manuscripts in Volume 12 (2015).

Erika Aaron

USA

Aniekan Abasiattai

Nigeria

Nurilign Abebe

Ethiopia

Gedefaw Abeje

Ethiopia

Saloua Abouchadi

Morocco

Roselline Achola

Uganda

Ishag Adam

Sudan

Abebaw Addis

Ethiopia

Temilayo Adeyeye

USA

\author{
Yohannes Adinew \\ Ethiopia
}

Jones Adjei

Canada

Gabriel Agboado
UK

Nadia Ahmad

United Arab Emirates

Baldreldeen Ahmed

Qatar

Sharon Ahumuza

Uganda

David Akeju

Nigeria

Moazzam Ali

Switzerland

Carmen Alvarez-Nieto

Spain

\section{Joshua Amo-Adjei \\ Ghana}

\section{Carukshi Arambepola}

Sri Lanka

\section{Emmanuel Asampong \\ Ghana}

José Ayres

Brazil

Syed Khurram Azmat Belgium

Syed Khurram Azmat
Pakistan

Syed Khurram Azmat

Canada

Qadeer Baig

Pakistan

Francis Bajunirwe

Uganda

\footnotetext{
*Correspondence: belizanj@gmail.com

${ }^{1}$ Institute for Clinical Effectiveness and Health Policy (IECS), Buenos Aires,

Argentina

Full list of author information is available at the end of the article
} 
Sudharsanam Balasubramaniam India

Justus Kafunjo Barageine

Uganda

Dana Barthel

Germany

Alka Barua

India

Hyam Bashour

Syrian Arab Republic

Hyam Bashour

Syria

Fereshteh Behmanesh

Iran

Regina Benevides

USA

Anne-Marie Bergh

South Africa

Jane Bertrand

USA

Ana Pilar Betran

Switzerland

Ana Pilar Betrán

Switzerland

Abdeldjellil Bezzaoucha

Algeria

Miteshkumar Bhanderi

India

Gashaw Biks

Ethiopia

Deborah Billings

USA

Jesper Bonde

Denmark

Ana Luiza Borges

Brazil

Andrea Borini

Italy

Loretta Brabin

UK

Giordana Braga

Brazil

Ricardo Cavalli

Brazil
Sue Cavill

UK

Nomita Chandhiok

India

Venkatraman Chandra-Mouli

Switzerland

Jeremiah Chikovore

South Africa

Antony Chikutsa

Zimbabwe

Livia Ciabati

Brazil

Jesse Clark

USA

John Cleland

UK

Kathya Lorena Cordova Pozo

Bolivia

Kevin Coughlin

Canada

Sienna Craig

USA

Doreen Crawford

UK

Eugene Kofuor Maafo Darteh Ghana

Dane De Silva

Canada

Adriane Delicio

Brazil

Zelmen Demeke

USA

Markos Desalegn

USA

Niveditha Devasenapathy India

Sangappa Dhaded

India

Jose Luis Diaz-Rossello

Uruguay

Jill Durocher

USA

Onyinye Edeh

Nigeria
Alison El Ayadi

USA

Sebastian Eliason

Ghana

Ifeanyichukwu Ezebialu

Nigeria

Fabio Facchinetti

Italy

Huma Farid

USA

Bukola Fawole

Nigeria

Gedefaw Fekadu

Ethiopia

Janise Ferreira

Brazil

Mario Philip Festin

Switzerland

Tamara Fetters

USA

Tabassum Firoz

Canada

Morenike Folayan

Nigeria

Florent Fouelifack

Cameroon

Ana Franzon

Brazil

Ana Galhardo

Portugal

Raman Gangakhedkar India

John Ganle

Ghana

Osvaldo Ulises Garay

Argentina

Abebaw Gebeyehu Worku

Ethiopia

Ewenat Gebrehanna

Ethiopia

Jillian Gedeon

Canada

Pamela Geller

USA 


Caitlin Gerdts
USA
Yoseph Gessesse
Ethiopia
Belaineh Girma
Ethiopia
Laura Goetzl
USA
Jessica Gorman
USA

Dereje Habte

Ethiopia

Gwyn Hainsworth

USA

Partha Haldar

India

Kelly Hallman

USA

Claudia Hanson

Sweden

Karen Hardee

USA

Abigail Harrison
USA

Emily Harville

USA

Abigail Hatcher

USA

Amanda Henry

Australia

Anna Hjelmstedt

Sweden

Johnny Holloway

USA

Lianne Holten

USA

Christopher Howson

USA

Patrick Idoko

Gambia

Chibuzo Ilonze

USA

Victor Inem

Nigeria
Roger Ingham

UK

Lindsey Jackson

USA

Kapila Jayaratne

Sri Lanka

Michael Joffe

UK

Nicole Johnson

USA

Tanya Jones

Australia

Allen Kabagenyi

Uganda

Frank Kaharuza

Uganda

Alexander Kalimbira

Malawi

Shashi Kant

India

Helle Karro

Estonia

Paul Kawale

Malawi

Nancy L Kerr

USA

Kavindra Kesari

Finland

Assaad Kesrouani

Lebanon

Omid Khorram

USA

Simon Peter Sebina Kibira

Uganda

Caron Kim

Switzerland

Theresa Kim

Canada

Rolf Klemm

USA

Thecla Kohi

Tanzania

Yacouba Kone

Mali
Theunis Kruger

South Africa

Lucy Kululanga

Malawi

Manisha Kumar

India

Lily C Kumbani

Malawi

Amos Laar

Ghana

Eve Lackritz

USA

Taavi Lai

Estonia

Cate Lane

USA

Evaline Lang'at

Kenya

Margareta Larsson

Sweden

Felicia Lester

USA

Magdalena Lewicka

Poland

Els Leye

Belgium

Cheng Li

China

Rupeng Li

USA

Yan Li

New Zealand

Gunilla Lindmark

Sweden

Jaime Lopez

USA

Beibei Lu

USA

Fernando Luiz Affonso Fonseca Brazil

Adriana Gomes Luz

Brazil

Moke Magoma

Tanzania 
Thulani Magwali

Zimbabwe

Fabiana Mamede

Brazil

Sudharsanam Manni Balasubramaniam

India

Abubakar Manu

Ghana

Donald Marazzo

USA

Mariana Martins

Portugal

Magdalena Mattebo

Sweden

Susannah Mayhew

UK

João Mazzoncini De Azevedo-Marques

Brazil

Anthony Mbonye

Uganda

Elizabeth Mcclure

USA

Sunil Mehra

India

Tekleab Mekbib

Ethiopia

Tekleab Mekbib

USA

Gustavo Mesch

Israel

Kara Michels

USA

Kristien Michielsen

Belgium

Deborah Mindry

USA

Suneeta Mittal

India

Iryna Mogilevkina

UKraine

Lamiya Mohiyiddeen

UK
Glen Mola

Papua New Guinea

Ilza Monteiro

Brazil

Ann Moore

USA

Alison Morgan

Australia

Olawale Moronkola

Nigeria

Leonardo Moscovici

Brazil

Kidza Mugerwa

Uganda

Stembile Mugore

USA

Wilson Muhwezi

Uganda

Saiqa Mullick

South Africa

Erik Munroe

UK

Fabien Munyaneza

Rwanda

Jane Namasasu

Malawi

Nehemiah Nando

Zimbabwe

Enock Ngome

Botswana

Jm Nicholson

USA

Dabere Nigatu

Ethiopia

Nik Daliana Nik Farid

Malaysia

Carolyne Njue

Kenya

Vicky Nogueira Pileggi

Brazil

Vicky Nogueira-Pileggi

Brazil
Linda Nyondo

Malawi

Francis Obare

Kenya

Mary Obiyan

Nigeria

Jeanne O'Brien

USA

Clifford Odimegwu

South Africa

Babasola Okusanya

UK

Funmilola Olaolorun

USA

Berend Olivier

Netherlands

Lemessa Oljira

Ethiopia

Comfort Olorunsaiye USA

Karen Pak Oppenheimer USA

Ngozi Orazulike

Nigeria

Katrien Oude Rengerink

Netherlands

Tia Palermo

Italy

Louis Peeters

Netherlands

Pascal Petitet

France

Sharon Phillips

USA

Cynthia Pileggi-Castro

Brazil

Torie Plowden

USA

Zahida Qureshi

Kenya

Sibylle Rahlenbeck

Germany 
Laila Rahman

Canada

Vibeke Rasch

Denmark

Krishna Regmi

UK

Tamar Renaud

USA

Jennifer Requejo

Switzerland

Heidi Reynolds

USA

Atif Riaz

Pakistan

Liesbeth Rijsdijk

Netherlands

Michael Ripple

USA

Narjis Rizvi

Pakistan

Nuriya Robinson

USA

Haleh Sangi-Haghpeykar

USA

Diane Santa Maria

USA

Belen Sanz Barbero

Spain

Katherine Sapra

USA

Karen Schliep

USA

Agumasie Semahegn

Ethiopia

H Senanayake

Sri Lanka

Carrie Shandra

USA

Gaurav Sharma

UK

Sumedha Sharma

Canada
Dorothy Shaw

Canada

Muhammad Ishaque Sheikh

Pakistan

Sana Sheikh

Pakistan

Chander Shekhar

India

Sonjelle Shilton

USA

Estelle Monique Sidze

Kenya

Robert Silver

USA

Maria Asuncion Silvestre

Philippines

Sara Simonsen

USA

Joao Souza

Brazil

Joao Paulo Souza

Brazil

Joao Paulo Souza

Switzerland

Ilene Speizer

USA

Chandrashekhar Sreeramareddy

Nepal

Joseph Stanford

USA

Rachel Steward

USA

Elizabeth Stringer

USA

Joar Svanemyr

Norway

Gunilla Sydsjo

UK

Bee Kang Tan

UK

Diane Taylor

USA
Danielle Teixeira

Brazil

Tesfalidet Tekelab

Ethiopia

Zelalem Teklemariam

Ethiopia

Marleen Temmerman

Switzerland

Britt Pinkowsky Tersbøl

Denmark

Gezahegn Tesfaye

Ethiopia

Stefanie Theuring

Germany

Myrte J Tielemans

Netherlands

Tizta Tilahun

Ethiopia

Aytekin Tokmak

Turkey

Sonila Tomini

Netherlands

Nnanna Ugwu

Nigeria

Vivian UKah

Canada

Priti Upadhyay

Nepal

Marcelo Urquia

Canada

Anke Van Der Kwaak

Netherlands

Carolina Vieira

Brazil

Elisabeth Vieira

Brazil

Joshua Vogel

Switzerland

Elisa Wells

USA

Paige Williams

USA 
Deborah Wing

USA

Eskinder Wolka

Ethiopia

Eileen Yam

USA

Kai Yao

USA
Tebikew Yeneabat

Ethiopia

Engida Yisma

Ethiopia

Nina Zamberlin

Argentina

Xiao-Ming Zhu

China

\section{Author details}

Institute for Clinical Effectiveness and Health

Policy (IECS), Buenos Aires, Argentina. ${ }^{2}$ Division of Intramural Population Health Research, of the

Eunice Kennedy Shriver National Institute of Child Health and Human Development, National Institutes of Health, Rockville, USA. ${ }^{3}$ Intensive Care Unit Department of Obstetrics and Gynecology, School of Medical Sciences, State University of Campinas, Campinas, Brazil. 